\title{
A Review of Progress in FDTD Maxwell's Equations Modeling of Impulsive Subionospheric Propagation Below $300 \mathrm{kHz}$
}

\author{
Jamesina J. Simpson, Student Member, IEEE, and Allen Taflove, Fellow, IEEE
}

\begin{abstract}
Wave propagation at the bottom of the electromagnetic spectrum (below $300 \mathrm{kHz}$ ) in the Earth-ionosphere waveguide system has been an interesting and important area of investigation for the last four decades. Such wave propagation is characterized by complex phenomena involving nonhomogeneous and anisotropic media, and can result in resonances of the entire Earth-ionosphere cavity. In the spirit of this Special Issue, the goal of this paper is to call attention to emerging finite-difference time-domain computational solutions of Maxwell's equations for wave propagation below $300 \mathrm{kHz}$ which promise to complement and extend previous analyses by pioneers such as Profs. Wait and Felsen. The following topical areas are discussed: long-range two-dimensional propagation, lightning sources and radiation, global propagation, Schumann resonances, hypothesized pre-seismic lithosphere sources and radiation, detection of deep underground resource formations, and remote sensing of localized ionospheric anomalies. We conclude with a prospectus for future research, especially in incorporating the physics of the anisotropic, nonhomogeneous magnetized plasma in a global planetary ionosphere.
\end{abstract}

Index Terms-Earth, earthquake, elves, extremely low frequency (ELF), finite-difference time-domain (FDTD), global temperature, ionosphere, ionospheric disturbances, lightning, low frequency (LF), oil fields, propagation, radar, remote-sensing, Schumann resonances, sprites, transient amplitude and phase perturbations of subionospheric signals (TRIMPIS), ultralow frequency (ULF), very low frequency (VLF), waveguide.

\section{INTRODUCTION}

W AVE propagation at the bottom of the electromagnetic (EM) spectrum in the Earth-ionosphere waveguide system, specifically at frequencies below $300 \mathrm{kHz}$, has been an interesting and important area of investigation for the last four decades. EM wave propagation within this system involves complex interactions with the lithosphere, oceans, and ionosphere, leading to resonances that involve literally the entire Earth-ionosphere cavity. Currently, propagation phenomena below $300 \mathrm{kHz}$ form the physics basis of remote-sensing investigations of lightning and sprites [1], [2], global temperature change [3], subsurface structures [4], [5], submarine communications [6], and potential earthquake precursors [7], [8].

Wave propagation below $300 \mathrm{kHz}$ within the waveguide bounded by the Earth's lithosphere and ionosphere, and more

Manuscript received February 24, 2006; revised September 2, 2006.

The authors are with the Electrical Engineering and Computer Science Department, Northwestern University, Evanston, IL 60208 USA (e-mail: j-simpson @northwestern.edu).

Color versions of one or more of the figures in this paper are available online at http://ieeexplore.ieee.org.

Digital Object Identifier 10.1109/TAP.2007.897138 generally about other planetary bodies, is characterized by complex phenomena involving nonhomogeneous and anisotropic media. In the spirit of this Special Issue, the goal of this paper is to call attention to emerging purely computational solutions of Maxwell's equations for wave propagation below $300 \mathrm{kHz}$ that promise to complement and extend previous analyses by pioneers such as Profs. J. R. Wait and L. B. Felsen. In fact, this paper can be viewed as an extension of a key theme presented in the 2002 Sevgi, Akleman, and Felsen article, "Groundwave propagation modeling: Problem-matched analytical formulations and direct numerical techniques," which appeared in the IEEE Antennas and Propagation Magazine dedicated to the memory of Prof. Wait [9]. Quoting from [9], Sevgi et al. endeavor "to trace the development of methodologies from the early, idealized, analytically tractable modeling [of wave propagation] to successively more realistic approaches, made possible by the availability of massive computational resources." They begin with analytical ray and mode techniques, progress to a frequency-domain algorithm, and conclude with their more recent finite-difference time-domain (FDTD)-based [10] wave propagator for signals above $1 \mathrm{MHz}$. Sevgi et al. conclude that the FDTD-based algorithm is "efficient and reliable for broadband (pulse) propagation," and that although FDTD can be computationally expensive, "the need for physics-based numerical algorithms is likely to continue."

In this paper, we shift to a frequency range much lower than that presented in [9] in order to highlight what Sevgi et al. termed "more realistic approaches" capable of taking advantage of "the availability of massive computational resources." We describe ambitious FDTD methodologies for modeling the Earth-ionosphere system, ranging from long-distance two-dimensional (2-D) propagation models to fully three-dimensional (3-D) global Earth-ionosphere system models. These algorithms exemplify emerging capabilities for simulating wave propagation below $300 \mathrm{kHz}$ in the complicated, inhomogeneous lithosphere and ionosphere of the Earth, as well as potentially other similar planetary environments.

This paper's organization is according to the following topical areas: long-range 2-D propagation, lightning sources and radiation, global propagation, Schumann resonances, hypothesized pre-seismic lithosphere sources and radiation, detection of deep underground resource formations, and remote sensing of localized ionospheric anomalies. We conclude with a prospectus for future research, especially in incorporating the physics of the anisotropic, nonhomogeneous magnetized plasma in a global planetary ionosphere. 


\section{LONG-RANGE 2-D PROPAGATION}

Berenger was the first to use the FDTD method to model subionospheric wave propagation at frequencies below 300 $\mathrm{kHz}$. His conference abstracts in this area date back to 1994 [11]. His work had two initial goals: 1) to compare FDTD results with previous frequency-domain mode theory calculations [12] and 2) to create an entirely new model having the capability of accommodating continuously varying parameters over the propagation path.

The basis for Berenger's first model is a 2-D spherical-coordinate grid extending from the Earth's surface upwards to the effective reflection height in the ionosphere at the frequency of interest, and extending a few thousand kilometer $(\mathrm{km})$ laterally along a great-circle cut. Perfect electric conductor (PEC) boundary conditions terminate the grid in all four directions, and a surface impedance is applied at the Earth's surface to model the finite conductivity of the lithosphere or ocean. Initially the natural geomagnetic field is not taken into account. However this is later modeled in [13] by way of solving the differential equations governing the current density in the ionosphere. The formulation of [14] achieves a gyrotropic (anisotropic) ionosphere model, a feature particularly important for nighttime propagation.

In [13]-[15], several techniques are developed to reduce the computational burden of modeling propagation over several megameters $(\mathrm{Mm})$. For example, in [13], Thevenot et al. introduce a technique involving a moving computational spatial domain extending backwards from the wavefront until steady state is attained. So long as reflections from the rear grid edge (opposite to the direction of interest) and propagation speeds are carefully considered, this technique can be used to significantly decrease the size of the grid.

A second way Berenger reduces the computational burden is by counteracting the numerical angular dispersion inherent to the FDTD mesh [14]. This strategy permits the use of coarser grids. That is, high-resolution meshes are needed for subionospheric propagation in the $3-300 \mathrm{kHz}$ range because "more than ten modes contribute significantly to the field strength" [14]. Each of these modes propagates with a different eigenangle, and therefore accrues a different phase shift at varying distances from the source. Berenger solves this issue by introducing an anisotropic permeability. Specifically, an artificial permeability $\mu_{r}$ is incorporated into the radial (vertical) derivatives of Maxwell's equations [14], [15]. This technique provides very good results for propagation at a single frequency or over a narrow range of frequencies, and for propagating modes having eigenangles within a certain range.

In his work, Berenger shows good agreement between waveguide mode theory and FDTD calculations for single-frequency propagation from a vertical dipole antenna. He considers typical daytime and nighttime ionosphere conductivity profiles, transitions between day and night, and disturbed conditions during a nuclear burst [13], [15]. Although Berenger finds that FDTD is more computationally demanding than mode theory, he notes that ongoing improvements in computer resources will continue to decrease simulation times in the future. Further, he states that FDTD "is more versatile than the waveguide method" since it can provide results for general wideband and impulsive applications [15].

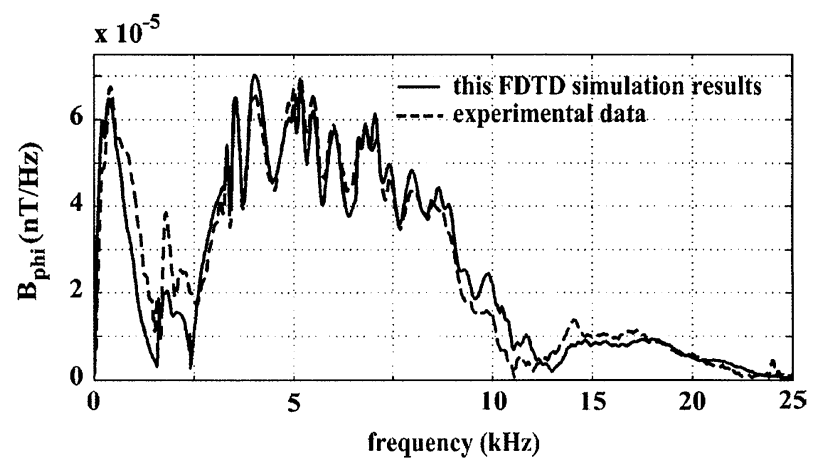

Fig. 1. Comparison up to $25 \mathrm{kHz}$ between experimental data collected by a sensor at Duke University with the 2-D cylindrical FDTD model predictions described in [17] (figure courtesy of [17]). The data shown is for 10 sferics radiated from negative lightning discharges at distances of about $629 \mathrm{~km}$.

\section{LIGHTNING SOURCES AND RADIATION}

We next consider FDTD modeling of lightning, the strongest natural source of waves in the $3 \mathrm{~Hz}-30 \mathrm{kHz}$ frequency range at the Earth's surface. In [16], Cummer compares FDTD results to mode theory and analytical calculations for propagation in this frequency band from a broadband lightning discharge over a 1000-km lossy ground path. For this study, he employs a 2-D cylindrical-coordinate FDTD grid having a PEC ground and an anisotropic but frequency-independent conducting ionosphere (assumes the collision frequency is much higher than the wave frequency at all altitudes).

Reference [16] reports "extremely good" agreement between numerical mode theory and FDTD for nighttime spectra below $10 \mathrm{kHz}$ radiated from the lightning source, and a comparable level of agreement for the daytime spectra. Reference [16] also finds that a "major strength of the FDTD technique... is that all of the fields (discharge and post-discharge, evanescent and propagating) are automatically calculated, while most other solution techniques are forced to treat these fields separately." These fields become increasingly important at frequencies below approximately $2 \mathrm{kHz}$ and distances of less than approximately $100 \mathrm{~km}$ from a lightning stroke. Further, FDTD can, in principle, permit straightforward modeling (with no increase in simulation time) of arbitrary horizontal as well as vertical inhomogeneities of the atmosphere and Earth. As a result, [16] concludes, "the simplicity of FDTD propagation modeling and ever-increasing computer power probably make FDTD the technique of the future."

In [17], Hu and Cummer improve the 2-D cylindrical FDTD model described in [16] by treating the ionosphere as a true cold plasma. Their new model includes the effects of charged particles (electrons, positive ions, and negative ions). It also includes an Earth's curvature correction, as well as a surface impedance boundary condition (SIBC) [18], [19] for modeling the lossy ground. As a result, the model of [17] provides improved accuracy to altitudes of up to $\sim 200 \mathrm{~km}$ and frequencies up to $\sim 30 \mathrm{kHz}$. Hu and Cummer validate the model of [17] by comparing FDTD calculations to mode theory solutions and broadband experimental data. Fig. 1 (courtesy of [17]) compares the

\footnotetext{
${ }^{1}$ We note here that these claims are strictly speaking applicable only to equivalent wavelengths that are adequately resolved by the FDTD grid.
} 
FDTD model results with experimental data for the frequency spectra of ten sferics radiated from negative lightning discharges at distances of about $629 \mathrm{~km}$. The level of agreement is "very strong" considering the number of uncertainties that prevent a better comparison [17].

Yang and Zhou [20] have also employed a 2-D cylindrical coordinate FDTD model to study EM fields very close to lightning channels. They find that the calculated vertical electric $(E)$-field agrees with measurements taken at $15 \mathrm{~m}$ distance. Further, the horizontal $E$-field at a distance of $100 \mathrm{~m}$ agrees with the Cooray-Rubinstein approximation [21], [22].

Lightning discharges have also been associated with complicated phenomena in the lower ionosphere that result in transient amplitude and phase perturbations of subionospheric signals (TRIMPIS), or transient perturbations (amplitude and phase) of subionospheric signals [23]. Two classes of TRIMPIS exist, both of which have been studied using FDTD, among other methods. The first class is known variously as lightning-induced electron precipitation (LEP) TRIMPIS, classic TRIMPIS, or whistler-induced electron precipitation (WEP) TRIMPIS. The second class, known as early TRIMPIS or early/fast TRIMPIS, are those assumed not caused by lightning-induced electron precipitation because of their very short onset delay after a lightning discharge. This second class includes sprites and elves.

Poussard and Corcuff [24] have studied the first class of TRIMPIS using the FDTD method. They employ 2-D FDTD models based on Berenger's initial propagation code [11]. However their 2-D FDTD model includes all six field components and no phase information. As a result, Poussard and Corcuff propose a hybrid system involving both FDTD and mode theory to study propagation below $10 \mathrm{kHz}$, and predict the location, longitudinal extent, and height of disturbed regions associated with the Trimpi events. Their results show good agreement with temporal signatures measured in France for signals arriving from England and the United States.

Otsuyama and Hayakawa [25] have studied the second class of TRIMPIS using the FDTD method. They use a 2-D FDTD model of a $500-\mathrm{km}$ long propagation path bounded by a PEC Earth and an ionosphere having an exponential conductivity profile. They first calculate scattering from sprite-induced plasma sheets of varying widths and distances and extending $20 \mathrm{~km}$ downwards from the ionosphere. Second, they calculate EM scattering from ionospheric perturbations associated with elves extending $10 \mathrm{~km}$ downwards from the ionosphere. These perturbations are assumed to be regions of increased ionization expanding outwards (radially) for $1.5 \mathrm{msec}$ to a total of $450 \mathrm{~km}$, followed by a decay of ionization expanding outwards at the same rate and over the same region. Results in [25] show that scattering at $40 \mathrm{kHz}$ by elves is significantly larger than those from sprites. In fact, the calculated scattering from sprites is so small the authors hypothesize that a weak perturbed region in the ionosphere extending laterally beyond the approximately 40-km diameter sprite (as observed by optical measurements) must exist.

Finally, specific FDTD techniques to model wave generation by lightning have been published. For example, Sarto creates in [26] an absorbing boundary condition specifically for when the space-cell size is several thousand times smaller than the minimum wavelength, such as for the case of lightning interaction with complex structures. Second, Berenger introduces in [27] a post-processing technique for removing most of the numerical anisotropy of the FDTD mesh when modeling propagation over large distances $(>5000 \mathrm{~km})$, thereby allowing coarser grids to be used. His previous single-frequency correction technique of [13] is not applicable to studies involving broadband lightning. He finds that the time required for post-processing is significantly less than the computational costs of using a finer mesh.

\section{Fully 3-D Studies of RAdiation AND LoNG-Distance PROPAGATION}

Hayakwa and Otsuyama [28], [29] have reported a global 3-D spherical-coordinate, latitude-longitude model of the Earthionosphere waveguide based upon fundamental work by Holland [30]. Their grid resolution is $250 \times 250 \times 2 \mathrm{~km}$, and they assume a PEC ground and typical exponential conductivity profiles for the ionosphere according to [30]. Because they use the same grid as Holland, their model is subject to increasing space-cell eccentricity upon approaching the poles due to converging lines of longitude.

In [28] and [29], Hayakwa and Otsuyama simulate a lightning discharge at the Equator having a double-exponential current time-waveform similar to that reported by Bruce and Golde [32]. The calculated radiated waveforms and wave impedances are compared at several distances (between 5 and $20 \mathrm{Mm}$ ) from the source to those predicted by previous analytical formulations [33], [34]. Further, the effect of modeling one-half of the Earth having a daytime ionosphere profile and the other half having a nighttime profile is investigated for both the symmetrical and more realistic tilted cases with respect to the North and South Poles [29].

Simpson and Taflove [35]-[37] have also constructed a global 3-D latitude-longitude spherical-coordinate FDTD model of the Earth-ionosphere waveguide below $1 \mathrm{kHz}$ based upon the fundamental work by Holland [30]. However, their model includes a means to reduce the eccentricity of the cells in the polar regions by a novel adaptive cell-combining technique applied to adjacent grid-cells in the east-west direction. This technique permits maintenance of the time-step at nearly the level allowed by the Courant stability condition for the square equatorial cells, yielding a greatly improved computational efficiency relative to conventional spherical-coordinate formulations.

Working towards a 3-D model, they began with a 2-D spherical-coordinate, transverse magnetic (TM) grid of the Earth's surface [35]. Simpson and Taflove used this model to track an impulsive circular cylindrical wave as it propagates radially outward from a filamentary current source having a Gaussian timewaveform. The radiated pulse travels completely around the Earth-sphere model and then propagates radially inward to the antipode. Results are shown for a lossless $1024 \times 512$ grid spanning the Earth-sphere [35]. A high degree of isotropy is demonstrated for numerical wave propagation within the model despite concurrent wave propagation through both simple, uniform grid regions near the Equator and more complicated polar regions having a nonuniform mesh. Further, they demonstrate the $180^{\circ}$ phase reversals of the $E$-field at the antipode first calculated 


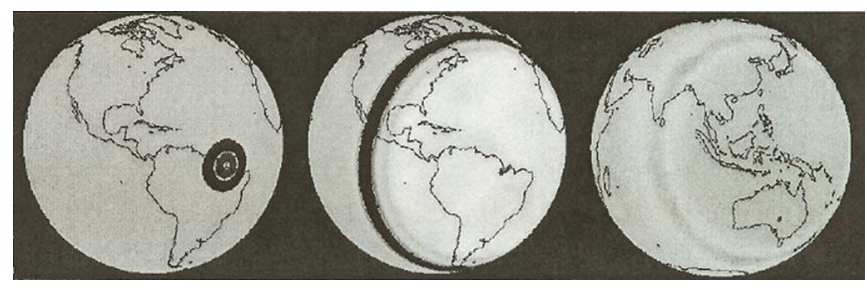

Fig. 2. Snapshots of impulsive wave propagation around the Earth as calculated by the 3-D FDTD model of [36] (the complete video can be downloaded at http://www.ece.northwestern.edu/ecefaculty/taflove/3Dmovietext@gif.avi) (figure courtesy of [36]).

by Wait [38], and a fundamental Earth-ionosphere-cavity resonance of $7.46 \mathrm{~Hz}$.

In [36], Simpson and Taflove expand the 2-D work of [35] to create a fully 3-D FDTD model of the entire Earth-ionosphere cavity extending between $\pm 100 \mathrm{~km}$ of sea level. Their 3 -D space lattice has a nominal resolution of $40 \times 40 \times 5 \mathrm{~km}$ at the Equator, and allows for variable cell size in the radial (vertical) direction. The East and West sides of the grid are connected to permit uninterrupted EM wave propagation around the Earth in the Equatorial direction. Only a single-processor laboratory computer with $2 \mathrm{~GB}$ of memory is needed to process the model.

For the 3-D latitude-longitude model of [36], Simpson and Taflove use topographic and bathymetric data from the NOAA-NGDC "Global Relief CD-ROM" [39]. Lithosphere conductivity is then assigned according to [40], depending upon whether the space lattice point is located directly below an ocean or within a continent. Isotropic ionospheric conductivity is assigned according to the exponential profile used in [41], which permits the most straightforward comparison of the present FDTD modeling results with the data reported in [42] since propagation is crucially affected by the ionosphere characteristics. However, FDTD models are capable of significantly greater ionospheric detail, i.e., day-to-night transitions, anisotropy, etc., than that possible using the analysis of [42] or other analytical methods.

To first validate their 3-D model, Simpson and Taflove excite the grid just off the Equator at $47^{\circ} \mathrm{W}$ with a vertical, $5-\mathrm{km}$ long current pulse having a Gaussian time-waveform. Fig. 2 (courtesy of [36]) shows snapshots of the global impulsive EM wave propagation radiated from the current pulse as calculated by their 3-D FDTD model. Their FDTD propagation modeling results are accurate to $\pm 1 \mathrm{~dB}$ over the range $50-500 \mathrm{~Hz}$.

Simpson and Taflove, in collaboration with climatologist R. Heikes, have also developed an alternative 3-D geodesic grid [43] of the global Earth-ionosphere cavity extending between $\pm 100 \mathrm{~km}$ of sea level [44]. This model employs hexagonal grid cells with a small, fixed number of pentagonal grid cells to span the spherical surface at each radial coordinate. As for the Simpson-Taflove latitude-longitude grid, they begin with a 2-D spherical-coordinate, TM geodesic grid of the Earth's surface [37], [45]. They then expand this 2-D work to create a fully 3-D FDTD geodesic grid allowing for variable cell size in the radial direction, and including details of the Earth's topography, bathymetry, and lithosphere and atmosphere conductivity values at a resolution of $\sim 63 \times 63 \times 5 \mathrm{~km}$ [44]. This grid is validated in the same manner as the latitude-longitude 3-D grid described above and in [36], with propagation modeling results accurate to within about $\pm 0.5 \mathrm{~dB} / \mathrm{Mm}$ in the frequency range of $50-500 \mathrm{~Hz}$.

The geodesic grid of [44] is superior to the previously reported Simpson-Taflove latitude-longitude grid of [36] in a number of respects. First, the geodesic grid completely avoids grid-cell convergences at the North and South Poles. Therefore, it provides much more isotropic wave propagation and is simpler to construct. Second, the geodesic grid ports efficiently to a massively parallel supercomputer. Third, it permits an easier interchange of data with state-of-the-art Earth-simulation codes used by the geophysics community.

\section{SCHUMANN RESONANCES}

In 1952, W. O. Schumann predicted the resonant frequencies of the Earth-ionosphere cavity below about $100 \mathrm{~Hz}$, which are now called Schumann resonances (SR) [46]. Interest in SRs have increased in recent years because of the apparent correlation between the resonant frequencies and global temperature changes [3], as well as with global lightning activity [47]. Further, SRs can be studied on other planetary bodies to provide information about their atmospheres [48]. To date, FDTD studies of SRs have been conducted by three groups, as described in the following text.

The first group, Otsuyama et al. [49], uses the global 3-D model described in [28], [29] and in Section IV to perform FDTD SR calculations. They simulate a lightning strike occurring at the Equator and record the $E$ and magnetic $(H)$ fields at distances between 5 and $20 \mathrm{Mm}$. Their results show that the amplitude and width of the SR peaks strongly depend on the source-observer distance. Further, they find that the FDTD-calculated SR peaks for the first and second modes are consistent with previous analytical calculations [31], [50].

The second group, Soriano et al. [51], use an azimuthally-symmetric 3-D spherical-coordinate FDTD model to conduct SR studies. Their model resolution is $1.5^{\circ}$ laterally and $5 \mathrm{~km}$ vertically, and they assume a PEC ground. First, a lossless Earth-ionosphere cavity is considered, with a PEC ionosphere boundary at a height of $60 \mathrm{~km}$. Good agreement is obtained between the FDTD-calculated resonances and previous analytical and transmission line matrix (TLM) results [52]. However, because the results differ from actual measurements, Soriano et al.. progress to a more advanced model including losses in the ionosphere. They model the quiet-atmosphere conductivity profiles of [52] and [53] to a height of $100 \mathrm{~km}$. The results obtained using this improved ionosphere model are in much better agreement with those of a semi-analytical two-scale height ionosphere model [54] and with actual measurements.

The third group, Yang and Pasko [55], also study SRs using initially an azimuthally-symmetric 3-D FDTD model of the Earth-ionosphere cavity. Their model extends to an altitude of $100 \mathrm{~km}$ with a resolution of $1000 \times 1000 \times 2.5 \mathrm{~km}$. They assume a PEC ground and test four different ionosphere conductivity profiles: 1) an ideal, free space cavity with a PEC ionosphere boundary; 2) a single-exponential profile with perturbation [56]; 3) a two-exponential profile using the same scale height for both sections [57], [54], [58]; and 4) a "knee" profile, 

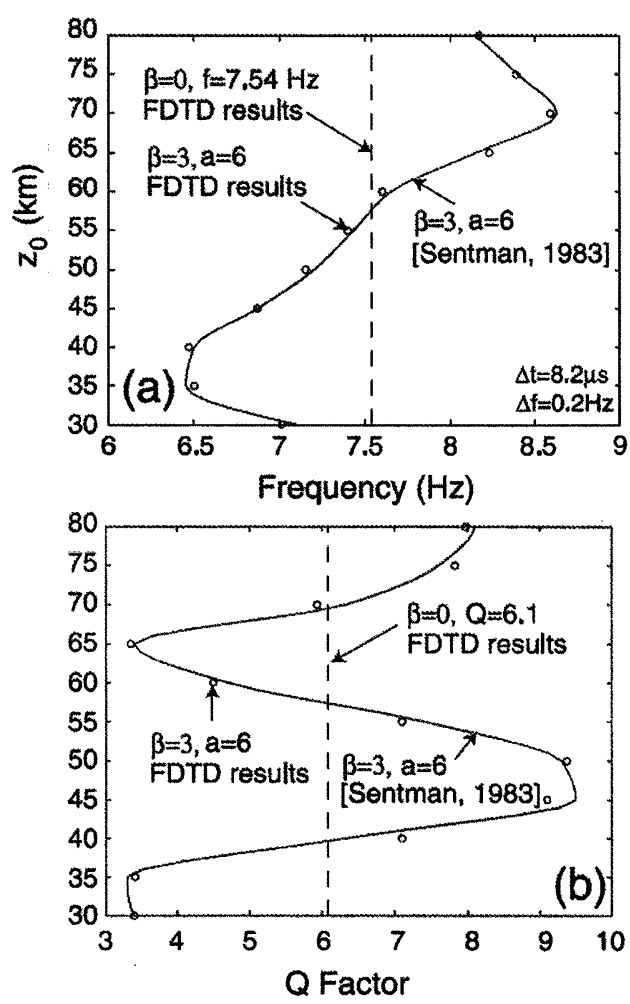

Fig. 3. Comparison between the first Schumann resonance (a) and $Q$ value (b) shifts as a function of altitude $\left(z_{0}\right)$ of the perturbation in the singleexponential ionosphere conductivity profile as predicted by the $3-\mathrm{D}$ azimuthally symmetric FDTD model described in [55] and the analytical (mode theory) results of [56] (figure courtesy of [55]).

e.g., [58]. Yang and Pasko excite their grid with a lightning current source and calculate the first five SR frequencies and associated Q factors from the radial $E$-field power spectrum at a distance of $2 \mathrm{Mm}$. Fig. 3 (courtesy of [55]) shows the predicted shifts in the first Schumann resonance (a) and $Q$ value (b) as a function of altitude $\left(\mathrm{z}_{0}\right)$ of the perturbation in the single-exponential ionosphere conductivity profile. Good agreement is obtained between the 3-D azimuthally symmetric FDTD model predictions described in [55] and the analytical (mode theory) results of [56].

In [55], Yang and Pasko also show that the "knee" profile provides the most accurate results compared to the theoretical model of Ishaq and Jones [59]. This observation agrees with that of [58], which models of the Earth-ionosphere system require an ionosphere having at least two scale heights in order to achieve realistic SR frequencies and Q-factors.

Additionally, Yang and Pasko study SRs for disturbed conditions associated with solar proton events and $\mathrm{X}$-ray bursts. The solar proton event is modeled as azimuthally symmetric conductivity perturbations at an altitude of $40 \mathrm{~km}$ and centered at the North and South Poles; the X-ray burst is modeled as an azimuthally symmetric conductivity perturbation at an altitude of $70 \mathrm{~km}$ and centered only at the North Pole. A discussion follows comparing the FDTD-calculated results with previous reports in the literature [56], [60]-[63].

Next, in [64], Yang and Pasko investigate diurnal and seasonal variations of the power of the first Schumann resonance. For this study, they use a fully 3-D FDTD model of the Earth-ionosphere waveguide (i.e., no longer azimuthally symmetric) having an ionospheric conductivity profile derived from the International Reference Ionosphere (IRI) [65] above $60 \mathrm{~km}$. The overall behavior of the calculated FDTD results agrees well with the experimental data of [66] for when the modeled lightning source location is varied between the three major lightning storm regions of the world (South-East Asia, Africa, South America) depending on the time of day, and the receiver is located in the Negev Desert, Israel [64].

Finally, in [67], Yang and Pasko investigate SRs on other planetary bodies, including Titan (Saturn's largest moon), Mars, and Venus. Understanding SRs on planetary bodies such as Titan could help support the existence of electrical discharges and provide other useful information regarding its lower atmosphere [67]. For their planetary FDTD models, conductivity profiles are derived from previously reported models. In [67], their FDTD results are compared to previously reported calculations, e.g., [48].

\section{Hypothesized Pre-Seismic Lithosphere Sources AND RADIATION}

Anomalous EM wave phenomena occurring prior to major earthquakes have been of particular interest for many years [68], [69]. A number of physical mechanisms related to hypothetical earthquake precursors have been proposed to explain the origin of such observations, including electrokinetic effects, piezoelectric effects, magnetohydrodynamic effects, charge generation processes, etc. [69]. However, the existence and characteristics of any pre-seismic EM signatures remain unclear and controversial.

In [70], Simpson and Taflove use their global 3-D FDTD model described in [36] and Section IV to model one of the possible mechanisms, electrokinetic currents, at depths of $2.5 \mathrm{~km}$ and $17 \mathrm{~km}$ near the hypocenter of the California 1989 Loma Prieta earthquake. They compare the FDTD-calculated surface $H$ field to analytical results and measurements previously reported in the literature [71]-[73]. Unlike previous analytical studies of this phenomena [71], [72], the full Maxwell's equations FDTD model accommodates the complete physics introduced by impulsive EM wave propagation through the conductive Earth, as well as EM wave reverberation due to round-the-world propagation. As a result, they obtain a significantly different temporal response at the Earth's surface than that of [71], [72]. Simpson and Taflove also report that their calculated spectra agree qualitatively with those measured by Fraser-Smith et al. [73] in the immediate hours prior to the earthquake.

\section{DETECTION OF DEEP UndERGROUND RESOURCE FORMATIONS}

Deep EM sounding of the Earth using controlled sources has been employed for decades as a means to determine the electrical properties of the Earth's lithosphere. Previously, controlled sources such as power lines, electrified railroads, and pulsed magnetohydrodynamic generators have been employed for such probing [74], [75]. However, the data obtained from these methods exhibits an unsatisfactory, wide variance [74]. 
Since the early 1990s a promising new system for global remote sensing at frequencies below $300 \mathrm{~Hz}$ has been under investigation [74], [75]. The advantages of using frequencies in this range for this application are: 1) a sufficiently large skin depth for deep probing (100 $\mathrm{m}$ in ocean and $10-15 \mathrm{~km}$ in crystalline shields); 2) low propagation attenuation, which could provide global remote sensing of the Earth with a single source; 3) good stability compared to higher frequency methods that are more influenced by ionospheric disturbances [74].

The sounding methodology described in [74] and [75], however, involves EM field impedance measurements to determine the resistivity of the underlying rock. This requires measurement of both the tangential $E$-field and the tangential $H$-field near the Earth's surface. In [44], [76], Simpson and Taflove propose a novel subsurface radar working at frequencies below $76 \mathrm{~Hz}$. This radar is designed to sense the presence of major oil deposits. It requires detection of only the radial $H$-field above the Earth's surface as a sinusoidal pulse is radiated outward from the former U.S. Navy Wisconsin Transmitting Facility (WTF). Signal acquisition and processing is facilitated by the well-characterized nature of the source and the proposed use of aerial surveys employing SQUID magnetometers [77].

To illustrate the operation of their proposed system, Simpson and Taflove report numerical simulations of radar returns from a hypothetical Alaskan oilfield excited by a pulsed $20-\mathrm{Hz}$ sinusoidal signal emitted from the WTF. Using their 3-D geodesic and latitude-longitude whole-Earth FDTD propagation models described in Section IV, [44], and [76], they show that detection of the radial (vertical) component of the scattered $H$-field exhibits an unexpected and very high degree of sensitivity to the presence of deeply buried conductivity anomalies of the lithosphere, much more so than the tangential $H$-field. They propose exploiting this phenomenon to establish a means to rapidly and inexpensively conduct aerial surveys of thousands of square $\mathrm{km}$ for significant oilfields.

\section{REMOtE SENSING OF LOCALIZED IONOSPHERIC ANOMALIES}

Despite substantial ground and satellite-based technology for studying the upper ionosphere, extracting the electron densities versus height in the D-region $(>95 \mathrm{~km})$ is still a difficult problem, particularly at night [78]. As a result, analysis and measurement of anomalous behavior of the D-region due to geophysical, solar, or other phenomena requires additional development.

Specifically, Wait [79] and Poulsen [80] published two of the first analyses of scattering from ionospheric perturbations. However, formulations such as these neglect mode conversion and thus do not apply to strong scatterers. Measurement techniques have included in-situ probing by rockets [81] and remote sensing of radiated spectra generated by random, naturally occurring sources, such as lightning [78]. However, these methods may not provide a practical means to continuously monitor the lower ionosphere and may be difficult or impossible to implement in many regions of the world.

In [82], Simpson and Taflove propose a novel radar working at $76 \mathrm{~Hz}$ for locating and characterizing localized ionospheric anomalies within $\sim 100 \mathrm{~km}$ of the Earth's surface. Their system assumes operation of the WTF as a distant, well-characterized, pulsed sinusoidal source, and passive detection of the resulting vertical $E$-field time-waveform at the Earth's surface in the vicinity of the ionospheric anomaly. Employing a man-made source such as the WTF could allow, in principle, a continuous and systematic monitoring scheme that avoids variabilities arising from naturally occurring sources such as lightning which have random properties.

To illustrate the operation of their proposed system, Simpson and Taflove [82] provide three calculations of vertical $E$-field signals at the Earth's surface in Los Angeles below bowl-shaped ionospheric conductivity depressions using their 3-D geodesicgrid whole-Earth FDTD wave propagation model described in [44] and Section IV. Unlike the analytical formulations of [79], [80], FDTD properly models mode conversion and thus applies to strong ionospheric scatterers. The depressions are assumed to have a depth of $20 \mathrm{~km}$ and a radius of either $100 \mathrm{~km}, 200$ $\mathrm{km}$, or $380 \mathrm{~km}$. Remote excitation is provided by a $76-\mathrm{Hz}$ sinusoidal pulse radiated essentially isotropically from the WTF. The FDTD-calculated results show that a simple measurement of the vertical $E$-field signal below a localized ionospheric depression can provide its location, size, shape, and depth. The radar proposed in [82] could therefore provide useful information regarding localized ionospheric depressions hypothesized to occur as earthquake precursors [83], as well as other local ionospheric conditions related to geophysical processes.

\section{CONClusions ANd ONGOING ReSEARCH}

In this review paper, we have highlighted emerging FDTD models of wave propagation at the bottom of the EM spectrum below $300 \mathrm{kHz}$ within the Earth-ionosphere system. Ranging from long-range 2-D propagation simulations to fully 3-D global propagation codes, these models complement and extend previous analyses by pioneers such as Profs. Wait and Felsen as summarized in [9]. The new FDTD models take advantage of the availability of large-scale computational resources to investigate much more complex EM wave interactions within the lithosphere, oceans, and ionosphere than was possible with previous analytical models. We have discussed applications of these FDTD models, including lightning sources and radiation, Schumann resonances, hypothesized pre-seismic lithosphere sources and radiation, detection of deep underground resource formations, and remote sensing of localized ionospheric anomalies. For the reader's convenience, Table I summarizes the principal FDTD techniques discussed in this paper, along with the most notable features and applications of each to date.

We conclude this paper with a prospectus for future research. One of the primary advantages of FDTD is its ability to model complex, continuously varying, nonhomogeneous and anisotropic media such as present in the ionosphere subject to the geomagnetic field. Thus, it would be particularly useful to extend the upper regions of existing 3-D global FDTD models well above the current height of approximately $100 \mathrm{~km}$ and include more details of the ionosphere to form a multiphysics simulator. Here, one possibility is to integrate available global data for the geomagnetic field [84] with FDTD codes that employ a magnetized plasma model of the ionosphere. A more ambitious possibility is to self consistently link global FDTD 
TABLE I

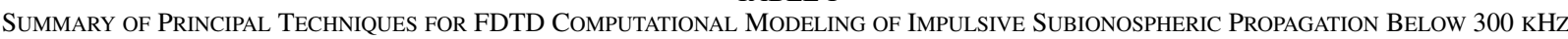

\begin{tabular}{|c|c|c|}
\hline Principal Techniques & $\begin{array}{c}\text { Notable Features } \\
\text { (to date) }\end{array}$ & $\begin{array}{l}\text { Applications } \\
\text { (to date) }\end{array}$ \\
\hline $\begin{array}{l}2 \text {-D great circle cut } \\
\text { propagation models }[11,13- \\
15,27]\end{array}$ & $\begin{array}{l}\text { Ionosphere anisotropy [13]; } \\
\text { surface-impedance ground } \\
\text { boundary [12]; isotropic } \\
\text { geomagnetic field [13]; } \\
\text { numerical dispersion } \\
\text { compensation [14]; moving } \\
\text { computational spatial domain } \\
\text { [13]; numerical anisotropy } \\
\text { removal [27] }\end{array}$ & $\begin{array}{l}\text { Long-distance propagation } \\
\text { modeling }[11,13-15,27] \text {; } \\
\text { nuclear burst }[15]\end{array}$ \\
\hline $\begin{array}{l}\text { 2-D azimuthally symmetric } \\
\text { models of the local Earth- } \\
\text { ionosphere waveguide } \\
\text { surrounding a lightning } \\
\text { channel }[16,17,20,24,25]\end{array}$ & $\begin{array}{l}\text { Cold plasma ionosphere [17]; } \\
\text { Earth curvature correction } \\
{[17]}\end{array}$ & $\begin{array}{l}\text { Propagation from lightning } \\
{[16,17,20] \text {; trimpis }} \\
\text { (whistlers, sprites, elves) }[24 \text {, } \\
25]\end{array}$ \\
\hline $\begin{array}{l}\text { 3-D azimuthally symmetric } \\
\text { models of the entire Earth- } \\
\text { ionosphere waveguide }[51,55 \text {, } \\
67]\end{array}$ & & $\begin{array}{l}\text { Schumann resonances [51, } \\
55] ; \text { x-ray bursts and solar } \\
\text { proton events [55]; }\end{array}$ \\
\hline $\begin{array}{l}\text { Fully 3-D latitude-longitude } \\
\text { models of the entire Earth- } \\
\text { ionosphere waveguide }[28,29 \text {, } \\
36,37,49,64,70,76]\end{array}$ & $\begin{array}{l}\text { Entire-Earth topography [36]; } \\
\text { spatially variable conductivity } \\
\text { of the lithosphere, oceans, and } \\
\text { ionosphere [36]; International } \\
\text { Reference Ionosphere (IRI) } \\
\text { model [64]; polar region cell- } \\
\text { combining technique [36]; } \\
\text { variable grid resolution in the } \\
\text { vertical (radial) direction [76] }\end{array}$ & $\begin{array}{l}\text { Propagation }[28,29,36,37] \\
\text { oil field radar [76]; preseismic } \\
\text { lithospheric current sources } \\
\text { [70]; Schumann resonances } \\
{[49,64] \text {; Schumann resonances }} \\
\text { on other planets [67] }\end{array}$ \\
\hline $\begin{array}{l}\text { Fully 3-D geodesic models of } \\
\text { the entire Earth-ionosphere } \\
\text { waveguide }[44,82]\end{array}$ & $\begin{array}{l}\text { Entire-Earth topography [44]; } \\
\text { spatially variable conductivity } \\
\text { of the lithosphere, oceans, and } \\
\text { ionosphere [44]; variable grid } \\
\text { resolution in the vertical } \\
\text { (radial) direction [44] }\end{array}$ & $\begin{array}{l}\text { Propagation [44]; radar for } \\
\text { ionospheric anomalies [82]; } \\
\text { oil field radar [44]; }\end{array}$ \\
\hline
\end{tabular}

simulators to sophisticated ionosphere models such as the U.S. Naval Research Lab's SAMI3 [85] or those listed at [86], [87]. Realization of combined electromagnetic wave/ionospheric physics models on a global scale would advance all of the applications discussed in this paper, and represent a fundamental advancement in geophysics.

\section{REFERENCES}

[1] L. Liebermann, "Extremely low-frequency electromagnetic waves 1. Reception from lightning," J. Appl. Phys., vol. 27, no. 12, pp. 1473-1476, 1956.

[2] S. A. Cummer, U. S. Inan, T. F. Bell, and C. P. Barrington-Leigh, "ELF radiation produced by electrical currents in sprites," Geophys. Res. Lett., vol. 25, p. 1281, 1998.

[3] E. R. Williams, "The Schumann resonance-A global tropical thermometer," Science, vol. 256, p. 1184, 1992.

[4] E. P. Velikhov, A. A. Zhamaletdinov, A. N. Shevtsov, A. D. Tokarev, Y. M. Kononov, L. B. Pesin, G. M. Kadyshevich, M. I. Pertel, and A. V. Veshchev, "Deep electromagnetic studies with the use of powerful ELF radio installations," Isvestiya - Phys. Solid Earth, vol. 34, no. 8, pp. 615-632, 1998.

[5] Y. B. Bashkuev and V. B. Khaptanov, "Deep radio impedance sounding of the crust using the electromagnetic field of a VLF radio installation," Izvestiya - Phys. Solid Earth, vol. 37, no. 2, pp. 157-165, 2001.

[6] J. R. Wait, "Project sanguine," Science, vol. 178, pp. 272-275, 1972.

[7] S. K. Park, M. J. S. Johnston, T. R. Madden, F. D. Morgan, and H. F. Morrison, "Electromagnetic precursors to earthquakes in the ULF band: A review of observations and mechanisms," Rev. Geophys., vol. 31, pp. 117-132, 1993.

[8] M. J. S. Johnston, "Review of electric and magnetic fields accompanying seismic and volcanic activity," Surv. Geophys., vol. 18, pp. $441-475,1997$.
[9] L. Sevgi, F. Akleman, and L. B. Felsen, "Groundwave propagation modeling: Problem-matched analytical formulations and direct numerical techniques," IEEE Antennas Propag. Mag., vol. 44, no. 1, pp. $55-75,2002$.

[10] A. Taflove and S. C. Hagness, Computational Electrodynamics: The Finite-Difference Time- Domain Method, 3 ed. Norwood, MA: Artech House, 2005.

[11] J. P. Berenger, "Finite-difference computation of VLF-LF propagation in the Earth-ionosphere waveguide," in EUROEM Symp., Bordeaux, France, May 29-Jun. 31994.

[12] R. A. Pappert and F. A. Ferguson, "VLF/LF mode conversion model calculations for air to air transmissions in the Earth-ionosphere waveguide," Radio Sci., vol. 21, no. 551, 1986.

[13] M. Thevenot, J. P. Berenger, T. Monediere, and F. Jecko, "A FDTD scheme for the computation of VLF-LF propagation in the anisotropic earth-ionosphere waveguide," Annals Telecommun., vol. 54, no. 5-6, pp. 297-310, 1999.

[14] J. P. Berenger, "Reduction of the angular dispersion of the FDTD method in the Earth-ionosphere waveguide," J. Electromagn. Waves Applicat., vol. 17, no. 8, pp. 1225-1235, 2002.

[15] J. P. Berenger, "FDTD computation of VLF-LF propagation in the Earth-ionosphere waveguide," Annals Telecommun., vol. 57, no. 11-12, pp. 1059-1090, 2002.

[16] S. A. Cummer, "Modeling electromagnetic propagation in the Earthionosphere waveguide," IEEE Trans. Antennas Propag., vol. 48, no. 9, pp. 1420-1429, 2000.

[17] W. Hu and S. A. Cummer, "An FDTD model for low and high altitude lightning-generated EM fields," IEEE Trans. Antennas Propag., vol. 54 , no. 5, pp. 1513-1522, 2007.

[18] J. G. Maloney and G. S. Smith, "The use of surface impedance concepts in the finite-difference time-domain method," IEEE Trans. Antennas Propag., vol. 40, pp. 38-48, 1992.

[19] S. Kellali, B. Jecko, and A. Reineix, "Implementation of a surface impedance formalism at oblique in FDTD method," IEEE Trans. Electromagn. Compat., vol. 35, pp. 347-356, 1993. 
[20] C. Yang and B. Zhou, "Calculation methods of electromagnetic fields very close to lightning," IEEE Trans. Electromagn. Compat., vol. 46, no. 1, pp. 133-141, 2004.

[21] V. Cooray, "Horizontal fields generated by return strokes," Radio Sci., vol. 27, pp. 529-537, 1992.

[22] M. Rubinstein, "An approximate formula for the calculation of the horizontal electric field from lightning at close, intermediate, and long range," IEEE Trans. Electromagn. Compat., vol. 38, pp. 531-535, 1996.

[23] H. J. Strangeways, "Lightning, TRIMPIS, and Sprites," in Review Radio Sci., 1993-1996, R. Stone, Ed. Oxford, U.K.: Oxford Univ. Press, 1996, pp. 741-780.

[24] A. M. Poussard and Y. Corcuff, "Numerical simulation of LEP TRIMPIS observed at Poitiers France, on signals from VLF transmitters," J. Atmos. Solar-Terres. Phys., vol. 62, pp. 207-224, 2000.

[25] T. Otsuyama and M. Hayakawa, "FDTD simulation and experimental result on VLF scattering by ionospheric perturbations in Earth-ionosphere waveguide," Trans. Inst. Elect. Eng. Jpn. A, vol. 122-A, no. 1, pp. 59-63, 2002.

[26] M. S. Sarto, "Innovative absorbing-boundary conditions for the efficient FDTD analysis of lightning-interaction problems," IEEE Trans. Electromagn. Compat., vol. 43, no. 3, p. 368, 2001

[27] J. P. Berenger, "Long-range propagation of lightning pulses using the FDTD method," IEEE Trans. Electromagn. Compat., vol. 47, no. 4, pp. 1008-1012, 2005.

[28] M. Hayakawa and T. Otsuyama, "FDTD analysis of ELF wave propagation in inhomogeneous subionospheric waveguide models," ACES J., vol. 17, no. 3, pp. 239-244, 2002.

[29] T. Otsuyama and M. Hayakawa, "FDTD Analysis of ELF wave propagation for realistic subionospheric waveguide models," Trans. Inst. Elect. Eng. Jpn. FM, vol. 124, no. 12, pp. 1203-1209, 2004.

[30] R. Holland, "THREDS: A finite-difference time-domain EMP code in 3-D spherical coordinates," IEEE Trans. Nucl. Sci., vol. NS-30, no. 6, pp. 4592-4595, 1983.

[31] A. P. Nickolaenko and M. Hayakawa, Resonances in the Earth-Ionosphere Cavity. Dordrecht: Kluwer Academic, 2002.

[32] C. E. R. Bruce and R. H. Golde, "The lightning discharge," J. Inst. Electr. Eng., vol. 88, pp. 487-505, 1941.

[33] A. P. Nickolaenko, M. Hayakawa, I. G. Kudintseva, S. V. Myand, and L. M. Rabinowicz, "ELF sub-ionospheric pulse in time domain," Geophys. Res. Lett., vol. 26, pp. 999-1002, 1999.

[34] A. P. Nickolaenko and M. Hayakawa, "Natural electromagnetic pulses in the ELF range," Geophys. Res. Lett., vol. 25, no. 16, pp. 3101-3106, 1998.

[35] J. J. Simpson and A. Taflove, "Two-dimensional FDTD model of antipodal ELF propagation and Schumann resonance of the Earth," IEEE Antennas Wireless Propag. Lett., vol. 1, no. 2, pp. 53-56, 2002.

[36] J. J. Simpson and A. Taflove, "Three-dimensional FDTD modeling of impulsive ELF propagation about the Earth-sphere," IEEE Trans. Antennas Propag., vol. 52, pp. 443-451, Feb. 2004.

[37] A. Taflove and J. J. Simpson, , A. Taflove and S. C. Hagness, Eds., "Introduction to Maxwell's equations and the Yee algorithm," in Computational Electrodynamics: The Finite-Difference Time-Domain Method, 3rd ed. Norwood, MA: Artech House, 2005.

[38] J. R. Wait, "Earth-ionosphere cavity resonances and the propagation of ELF radio waves," Radio Sci., vol. 69D, p. 1057, 1965.

[39] Global Relief CD-ROM NOAA-NGDC.

[40] J. Hermance, "Electrical conductivity of the crust and mantle," Global Earth Physics: A Handbook of Physical Constants, 1995, AGU.

[41] P. Bannister, "The determination of representative ionospheric conductivity parameters for ELF propagation in the Earth-ionosphere waveguide," Radio Sci., vol. 20, no. 4, pp. 977-984, 1985.

[42] P. Bannister, "ELF propagation update," IEEE J. Oceanic Eng., vol. 0E-9, no. 3, pp. 179-188, 1984.

[43] D. A. Randall, T. D. Ringler, and R. P. Heikes, "Climate modeling with spherical geodesic grids," Computing Sci. Eng., vol. 4, no. 5, pp. 32-41, Sep./Oct. 2002.

[44] J. J. Simpson, R. P. Heikes, and A. Taflove, "FDTD modeling of a novel ELF radar for major oil deposits using a three-dimensional geodesic grid of the Earth-ionosphere waveguide," IEEE Trans. Antennas Propag., vol. 54, no. 6, pp. 1734-1741, 2007.

[45] J. J. Simpson and A. Taflove, "Efficient modeling of impulsive ELF antipodal propagation about the Earth sphere using an optimized twodimensional geodesic FDTD grid," IEEE Antennas Wireless Propag. Lett., vol. 3, no. 11, pp. 215-218, 2004.
[46] W. O. Schumann, "On the radiation free self-oscillations of a conducting sphere, which is surrounded by an air layer and an ionospheric shell [in German]," Zeitschrift fuer Naturforschung, vol. 7a, pp. 149-154, 1952.

[47] C. Polk, , Coronoti and Hughes, Eds., "Relation of ELF noise and Schumann resonances to thunderstorm activity," in Planetary Electrodynamics. New York: Gordon and Breach, 1969, vol. 2, pp. 55-83.

[48] O. Pechony and C. Price, "Schumann resonance parameters calculated with a partially uniform knee model on Earth, Venus, Mars, and Titan," Radio Sci, vol. 39, no. 5, 2004.

[49] Y. Ando, M. Hayakawa, A. V. Shvets, and A. P. Nickolaenko, "Finite difference analyses of Schumann resonance and reconstruction of lightning distribution," Radio Sci., vol. 38, no. 6, p. 1103, 2003.

[50] D. D. Sentman, , H. Volland, Ed., "Schumann resonances," in Handbook of Atmospheric Electrodynamics. Boca Raton, FL: CRC Press, 1995, vol. 1, pp. 267-310.

[51] A. Soriano, E. A. Navarro, D. L. Paul, J. A. Porti, J. A. Morente, and I. J. Craddock, "Finite-difference time domain simulation of the Earthionosphere resonant cavity: Schumann resonances," IEEE Trans. Antennas Propag., vol. 53, no. 4, pp. 1535-1541, Apr. 2005.

[52] J. Morente, J. Molina-Cuberos, J. Porti, B. Besser, A. Salinas, K. Schwingenschuch, and H. Litchengger, "A numerical simulation of Earth's electromagnetic cavity with the transmission line matrix method: Schumann resonances," J. Geophys. Res., vol. 108, no. A5, 2003.

[53] K. Schlegel and M. Fuellekrug, "Schumann resonance parameter changes during high-energy particle precipitation," J. Geophys. Res, vol. 104, no. A5, pp. 10111-10118, 1999.

[54] D. D. Sentman, "Approximate Schumann resonance parameters for two-scale height ionosphere," J. Atmos. Terr. Phys., vol. 52, no. 1, pp. 35-46, 1990.

[55] H. Yang and V. P. Pasko, "Three-dimensional finite difference time domain modeling of the Earth-ionosphere cavity resonances," Geophys. Res. Lett., vol. 32, p. L03114, 2005.

[56] D. D. Sentman, "Schumann resonance effects of electrical conductivity perturbations in an exponential atmospheric/iononspheric profile," $J$. Atmos. Terr. Phys., vol. 45, no. 1, pp. 55-65, 1983.

[57] C. Greifinger and P. Greifinger, "Approximate method for determining ELF eigenvalues in the Earth-ionosphere cavity," Radio Sci, vol. 13, p. $831,1978$.

[58] V. C. Mushtak and E. Williams, "ELF propagation parameters for uniform models of the Earth-ionosphere waveguide," J. Atmos. Sol. Terr. Phys., vol. 64, 1989.

[59] M. Ishaq and D. L. Jones, "Method of obtaining radiowave propagation parameters for the Earth-ionosphere duct at ELF," Electron. Lett., vol. 13, p. 254, 1977.

[60] K. Schlegel and M. Fuellekrug, "Schumann resonance parameter changes during high-energy particle precipitation," J. Geophys. Res., vol. 104, p. 10, 111, 1999

[61] V. C. Roldugin, Y. P. Maltsev, G. A. Petrova, and A. N. Vasiljev, "Decrease of the first Schumann resonance frequency during solar proton events," J. Geophys. Res., vol. 106, p. 18,555, 2001.

[62] V. C. Roldugin, Y. P. Maltsev, A. N. Vasiljev, A. V. Shvets, and A. P. Nikolaenko, "Changes of Schumann resonance parameters during the solar proton event of 14 July 2000," J. Geophys. Res., vol. 108, no. A3, p. 1103, 2003.

[63] V. C. Roldugin, Y. P. Maltsev, A. N. Vasiljev, A. Y. Schokotov, and G. G. Belyajev, "Schuamnn resonance frequency incrase during solar X-ray bursts," J. Geophys. Res., vol. 109, no. A01216, 2004.

[64] H. Yang and V. Pasko, "3-D FDTD modeling of the variations of the Schumann resonance parameters in the Earth-ionosphere cavity," in Proc. USNC/URSI Nat. Radio Science Meeting, Albuquerque, NM, Jul. 2007, p. 723.

[65] D. Bilitza, "IRI 2000," Radio Sci., vol. 36, no. 2, pp. 261-276, 2001.

[66] Price and Melnikov, "Diurnal, seasonal, and inter-annual variations of the Schumann resonance parameters," J. Atmos. Solar-Terr. Phys., vol. 66, no. 13-14, pp. 1179-1185, 2004

[67] H. Yang, V. P. Pasko, and Y. Yair, "Three-dimensional finite difference time domain modeling of the Schumann resonance parameters on Titan, Venus, and Mars," Radio Sci., vol. 1, no. 2, p. RS2S03, Sep. 2006.

[68] S. K. Park, M. J. S. Johnston, T. R. Madden, F. D. Morgan, and H. F. Morrison, "Electromagnetic precursors to earthquakes in the ULF band: A review of observations and mechanisms," Rev. Geophys., vol. 31, pp. 117-132, 1993.

[69] M. J. S. Johnston, "Review of electric and magnetic fields accompanying seismic and volcanic activity," Surv. Geophys., vol. 18, pp. 441-475, 1997. 
[70] J. J. Simpson and A. Taflove, "Entire-Earth FDTD Modeling of Electrokinetic Effects Prior to the Loma Prieta Earthquake," Geophys. Res. Lett., vol. 32, p. L09302.

[71] M. A. Fenoglio, M. J. S. Johnston, and J. D. Byerlee, "Magnetic and electric fields associated with changes in high pore pressure in fault zones: application to the Loma Prieta ULF emissions," J. Geophs. Res., vol. 100, pp. 12951-12958, 1995.

[72] O. Majaeva, Y. Fujinawa, and M. E. Zhitomirsky, "Modeling of nonstationary electrokinetic effect in a conductive crust," J. Geomag. Geoelectr., vol. 49, pp. 1317-1326, 1997.

[73] A. C. Fraser-Smith, A. Bernardi, P. R. McGill, M. E. Ladd, R. A. Helliwell, and O. G. Villard Jr., "Low-frequency magnetic field measurements near the epicenter of the Ms 7.1 Loma Prieta earthquake," Geophys. Res. Lett., vol. 17, pp. 1465-1468, 1990.

[74] E. P. Velikhov, A. A. Zhamaletdinov, A. N. Shevtsov, A. D. Tokarev, Y. M. Kononov, L. B. Pesin, G. M. Kadyshevich, M. I. Pertel, and A. V. Veshchev, "Deep electromagnetic studies with the use of powerful ELF radio installations," Isvestiya, Phys. Solid Earth, vol. 34, no. 8, pp. 615-632, 1998.

[75] Y. B. Bashkuev and V. B. Khaptanov, "Deep radio impedance sounding of the crust using the electromagnetic field of a VLF radio installation," Izvestiya - Phys. Solid Earth, vol. 37, no. 2, pp. 157-165, 2001.

[76] J. J. Simpson and A. Taflove, "Whole Earth FDTD modeling of ELF electromagnetic sounding of oil deposits with the U.S. Navy's ELF transmitter system," in Proc. USNC/URSI Nat. Radio Science Meeting, Washington, DC, Jul. 3 - 82005.

[77] J. B. Lee, D. L. Dart, R. J. Turner, M. A. Downey, A. Maddever, G. Panjkovic, C. P. Foley, K. E. Leslie, R. Binks, C. Lewis, and W. Murray, "Airborne TEM surveying with a SQUID magnetometer sensor," Geophys., vol. 67, no. 2, pp. 468-477, Mar.-Apr. 2002.

[78] S. A. Cummer, U. S. Inan, and T. F. Bell, "Ionospheric D region remote sensing using VLF radio atmospherics," Radio Sci., vol. 33, pp. $1781-1792,1998$.

[79] J. R. Wait, "On phase changes in very low frequency propagation induced by an ionospheric depression of finite extent," J. Geophys. Res., vol. 69, p. 441, 1964.

[80] W. L. Poulsen, T. F. Bell, and U. S. Inan, "3-dimensional modeling of subionospheric VLF propagation in the presence of localized D-region perturbations associated with lightning," J. Geophys. Res.-Space Phys., vol. 95, no. A3, pp. 2355-2366, Mar. 1990.

[81] C. F. Sechrist Jr., "Comparisons of techniques for measurement of D-region electron densities," Radio Sci., vol. 9, p. 137, 1974.
[82] J. J. Simpson and A. Taflove, "ELF radar system proposed for localized D-region ionospheric anomalies," IEEE Geosci. Remote Sensing Lett., to be published.

[83] S. Pulinets and K. Boyarchuk, Ionospheric Precursors of Earthquakes. Berlin, Germany: Springer, 2004, p. 315.

[84] [Online]. Available: http://www.ngdc.noaa.gov/seg/geomag/models. shtml

[85] [Online]. Available: http://www.nrl.navy.mil/content.php?P=04REVIEW105

[86] [Online]. Available: http://www.ngdc.noaa.gov/stp/IONO/models.html

[87] [Online]. Available: http://modelweb.gsfc.nasa.gov/ionos/ionos_ index.html

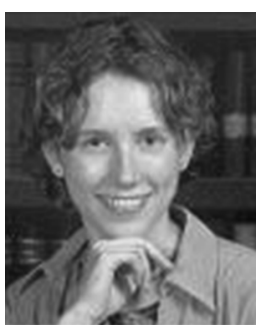

Jamesina J. Simpson ( $\left.\mathrm{S}^{\prime} 01\right)$ is a Ph.D. candidate in electrical engineering at Northwestern University, Evanston, IL.

Since her freshman undergraduate year, she has been a Research Assistant in Prof. Taflove's Computational Electromagnetics Laboratory. Her current research interests include FDTD modeling of geophysically induced extremely low-frequency propagation about the entire Earth and FDTD modeling of novel ultrahigh-speed bandpass wireless interconnects for next-generation digital circuits.

Ms. Simpson is a member of Tau Beta Pi and has received the National Science Foundation Graduate Research Fellowship, Walter P. Murphy Fellowship, IEEE AP-S Graduate Research Award, IEEE MTT-S Graduate Research Award, and the Richter Fellowship for her graduate studies.

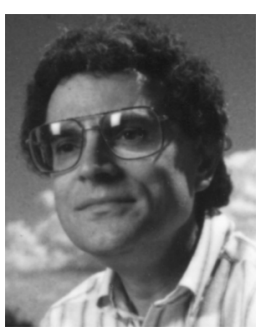

Allen Taflove (F'90) is a Professor in Northwestern University's EECS Department. He has helped to pioneer FDTD algorithms and applications since 1971. His publications include 115 journal papers and three editions $(1995,2000$, and 2005) of the book, Computational Electrodynamics: The Finite-Difference Time-Domain Method, which has become a standard reference in the FDTD field. He is listed by the Institute for Scientific Information as one of the most cited technical authors in the world. 